\title{
The Cambodian picture becomes clearer
}

IN the past few weeks, the people of many countries have been exposed to the plight of Cambodia, or Kampuchea, through television programmes, through dramatic gestures, such as Mrs Carter's visit to Thailand, and through the widespread publicity of the relief agencies. The response has been generous; governments have pledged all manner of aid, and voluntary bodies are receiving a steady flow of funds. There is an evident need for the care of many thousands of Khmer refugees in Thailand. But as more is learnt about the situation within Cambodia, it is emerging that the need is not so much for immediate, massive emergency aid to relieve a national famine (for which there is little evidence), but for steady aid over the medium and long term to bring a once flourishing country back to health.

Observers who have recently been in Cambodia, or who have worked amongst the refugees in Thailand, are now beginning to bring back a more comprehensive picture, and this is augmented by satellite photographs of areas under cultivation. As far as food is concerned, it is likely that a substantial harvest was available at the end of 1978. Most of the country has one harvest per year, so food resources under normal circumstances should have been fairly adequate for most of 1979 . Of course the circumstances are not normal and much of the stored rice could have been lost; possibly upwards of 200,000 tonnes of food aid has been shipped in this year at the request of the government but this should be put in the context of an annual consumption of about 1 million tonnes (assuming a population of 4.5 million). Where simple indicative tests such as the measurement of mid-upper arm circumference of children have been carried out, they equally suggest moderate under-nutrition rather than starvation.

The mid-term food supply in Cambodia depends very much on the 1979 harvest, which runs from October to December. Estimates of the amount of land under cultivation after the disruptions of the past year vary greatly, but the figures that are emerging suggest that less than a third of all arable land will be productive, and that the shortfall in food supplies for 1980 will be up to $50 \%$; pledges through the World Food Programme and from individual countries will ensure that this deficit will be made up for the next six months at least, provided that movement through ports of entry can be expedited.

The problem on the medical side is, if anything, more serious. It is reported that malaria is killing widely, and that untreated gastro-enteritis, tuberculosis and anthrax are also taking a heavy toll, although figures are proving hard to come by. During the dry season (until April) the incidence of malaria will fall, but concern is being expressed about the degree of drug-resistance being shown. Medical supplies have poured in beyond the capacity of local staff to use them and the problem for the immediate future is the development of a proper medical infrastructure to provide health care. With the (literal) decimation of the professional classes in Cambodia, no real progress towards self-sufficiency is likely until new schools are built, new people are tained and selfconfidence is re-established.

The real need for Cambodia is not to relieve a nationwide famine; it is to supply food, medical aid and transport steadily and intelligently over the coming months, and for a large scale investment both in rural and urban re-construction and in the rebuilding of the professions. This poses difficult questions for the more thoughful of those connected with relief. Film of starving children (and it cannot be denied that some children, particularly amongst the displaced, are starving), mention of widespread famine and hints of many further deaths in the coming weeks undoubtedly opens the floodgates of goodwill, but it also leaves relief agencies with strong political and emotional pressures to act on an emergency basis, even when it were better that investments were delayed until more information became available. There is a very serious dilemma here. Fund raisers know full well that tragic pictures bring in the money, but fund-spenders know equally well that the real needs are often elsewhere, or over a different time-frame, or for a different purpose. They know, for instance, that any money given now to alleviate the effects of the severe drought which has hit parts of India this year is ten times more valuable than cash that will flow in the summer of 1980 when the effects become all too visible.

The only resolution of this dilemma is slow, patient education of the public on the value of prevention. It is something that governments themselves should take on, if they see any merit at all in scientific assessments of disasters. The British government now winding down its development education programme which had only been given a breath of life a year ago - could well make an imaginative step. 